\title{
Altered Profile of Fecal Microbiota of Patients with Colon Diverticulitis
}

\author{
SHIN WATANABE*1) 2), HIKARU AKIZUKI*1), NORITOSHI YOSHIDA*1), \\ YOSHIYUKI MORI*1), ATSUSHI IHARA*1) \\ *1) Department of Surgery, Asakusa Hospital, Tokyo, Japan, \\ *2) Department of Microbiome Research, Juntendo University Graduate School of Medicine, Tokyo, Japan
}

\begin{abstract}
Objective: During colonoscopies, clinicians often find feces accumulated in the colonic diverticula. We hypothesized that these feces and potential changes in fecal microbial communities contribute to colon diverticulitis. The aim of this study was to investigate potential changes in the fecal microbiota in symptomatic uncomplicated diverticular disease by terminal restriction fragment length polymorphism (T-RFLP) analyses of fecal microbiota from patients with colonic diverticula, and stool samples. Materials: Fifteen patients with colon diverticulum and 28 healthy volunteers were enrolled. The fecal samples were classified as follows: Group I, feces from a healthy individual; Group II, feces in colonic diverticula obtained during colonoscopy; Group III, feces from the natural defecation of Group II patients.
\end{abstract}

Methods: Fecal microbiota profiles were evaluated by T-RFLP analysis.

Results: T-RF patterns of fecal microbiota were divided into three clusters. Most Group I samples were included in clusters A and B, whereas most Group II samples were included in cluster C. Operational taxonomic units of $657 \mathrm{bp}(\mathrm{p}<0.05)$ and $955 \mathrm{bp}$ $(\mathrm{p}<0.01)$ differed in abundance between patients with colon diverticulum and healthy individuals.

Conclusions: T-RFLP analyses revealed that the fecal microbial communities in patients with diverticular disease differed from those of healthy individuals, particularly for the operational taxonomic units of 657 and $955 \mathrm{bp}$. Changes in the fecal microbiota, including Lactobacillales and Clostridium subcluster XIVa, may play a role in diverticular disease.

Key words: colon diverticulum, microbiota, T-RFLP, Lactobacillales, Clostridium

\section{Introduction}

Approximately $20 \%$ of patients with diverticulosis will experience inflammatory complications of the disease, ranging in severity from a single mild acute attack of diverticulitis to more severe attacks characterized by perforation and abscess formation ${ }^{1)}$. Aging and reduced dietary fiber intake are the main pathological factors in this disease ${ }^{2)}{ }^{3)}$. The pathophysiology of diverticulitis involves an imbalance in colonic microbiota ${ }^{4)}{ }^{5}$. During colonoscopy, feces are often found accumulated in the colonic diverticula. These feces differ in appearance or solidity from those in the main intestinal tract. Thus, we hypothesized that the microbiota of feces in the diverticula may differ from that of normal feces and cause diverticulitis.

Terminal restriction fragment length polymorphism (T-RFLP) analysis enables the assessment of bacterial community structure and ecosystem diversity ${ }^{6}$. Research findings obtained by T-RFLP are consistent with those obtained by next-generation sequencing (NGS), which is now widely used ${ }^{7)}$. In the current study, we investigated changes in the diversity of fecal microbial communities in the colon diverticula of patients with colon diverticulitis by

Corresponding author: Shin Watanabe

Department of Surgery, Asakusa Hospital

2-26-15 Imado, Taito-ku, Tokyo 111-0024, Japan

TEL: +81-3-3876-1711 FAX: +81-3-3871-3626 E-mail: snwatana@juntendo.ac.jp

〔Received Mar. 5, 2019]〔Accepted June 21, 2019〕

J-STAGE Advance published date: Aug. 20, 2019

Copyright (C) 2019 The Juntendo Medical Society. This is an open access article distributed under the terms of Creative Commons Attribution License (CC BY), which permits unrestricted use, distribution, and reproduction in any medium, provided the original source is properly credited. doi: 10.14789/jmj.2019.65.JMJ19-OA07 
T-RFLP.

\section{Materials and Methods}

\section{Participants and method}

This study was a pilot case-control study carried out between December 2013 and November 2015. Fifteen patients who were diagnosed as having colon diverticulitis by computed tomography within the past 6 months, and were found to have several right-sided colonic diverticula containing impacted feces by colonoscopy were included in this study. We performed colonoscopies to collect feces accumulated in the right-sided colonic diverticula with biopsy forceps, and all patients who underwent colonoscopy provided a natural defecation specimen for analysis on the same day. As a control, healthy volunteers also provided natural defecation samples. All samples were stored in $100 \mathrm{mM}$ Tris-HCL ( $\mathrm{pH} \mathrm{9),} 40 \mathrm{mM}$ ethylenediaminetetraacetic acid, $4 \mathrm{M}$ guanidine thiocyanate, and $0.001 \%$ bromothymol, immediately. Exclusion criteria included a history of or current colorectal cancer, a history of or current chemotherapy or radiation therapy, and current use of antibiotics, probiotics or immunosuppressants. Fecal microbiota profiles were evaluated by T-RLP.

The fecal samples were classified as follows: Group I, stool samples from healthy individuals; Group II, samples of feces accumulated in the colonic diverticula; Group III, natural defecation samples from Group II patients. All procedures used in this study were approved by the ethical committee of Asakusa Hospital (Approval number 190007). Written informed consent was obtained from the patients for inclusion in this study and for the publication of this report and its accompanying images.

\section{Extraction of DNA}

Using the fluorescently labeled 516 forward primer (5'-TGCCAGCAGCCGCGGTA-3') and 1492 reverse primer (5'-GGTTACCTTGTTACGACTT3 '), the 16S rRNA genes were amplified from fecal DNA. The 5 ' end of the forward primers was labeled with 6-carboxyfluorescein (6-FAM), which was synthesized using a MultiScreen $\mathrm{PCR}_{96}$ filter plate (Millipore, Tokyo, Japan). The PCR amplification of DNA samples was performed according to the protocol described by Nagashima et $a l^{77}$. The purified polymerase chain reaction products $(3 \mu l)$ were digested with $10 \mathrm{U}$ of $B s I \mathrm{I}$ (New England Biolabs Inc., Ipswich, MA, USA) at $55^{\circ} \mathrm{C}$ for $3 \mathrm{~h}$. The $\mathrm{T}-\mathrm{RF}$ lengths were determined using an $\mathrm{ABI}$ PRISM 3130xl Genetic Analyzer (Applied Biosystems, Tokyo, Japan) in GeneScan mode.

\section{T-RFLP analysis}

$\mathrm{T}$-RF patterns were classified into 29 operational taxonomic units (OTUs) in accordance with the Nagashima method ${ }^{7)}$ ). Individual OTUs were quantified as a percentage of the total OTU area, expressed as a percentage of the area under the curve (\%AUC), which indicates the predominance of the different microorganisms that comprise each peak $^{6)}$. Bs II-digested T-RFLP profiles with predicted bacterial species were evaluated (TechnoSuruga Laboratory Co., Ltd., Shizuoka, Japan). Cluster analyses were performed, and a dendrogram of the T-RFLP profiles was generated by applying Ward's method. In this study, we focused on OUT of $657 \mathrm{bp}$ including Lactobacillales, which is strongly associated with irritable bowel syndrome (IBS), and OUT of $956 \mathrm{bp}$ reported to decrease in inflammatory bowel disease cases ${ }^{9}$.

\section{Statistical analysis}

The T-RF peak patterns were compared between patients with colon diverticulitis and healthy individuals using the Wilcoxon rank sum test. The canonical judgment function coefficient was obtained from discriminant analysis of the three groups. Each OTU was compared among the three groups using one-way analysis of variance (ANOVA). Differences with a $\mathrm{p}^{-}$value of less than 0.05 were regarded as significant. The data were analyzed using SPSS (IBM SPSS Statistics 20.0, Tokyo, Japan).

\section{Results}

Twelve men and three women, mean age 70 years [range: 60-77 years], were examined. Of these patients, six (40\%) also had diverticula in the left-sided colon. Multiple diverticula were defined as three or more diverticula in the right-sided colon. To prevent constipation, most patients had been taking oral magnesium oxide. None of the patients had a history of colorectal cancer or severe cardiovascular disease. Feces from 28 healthy 


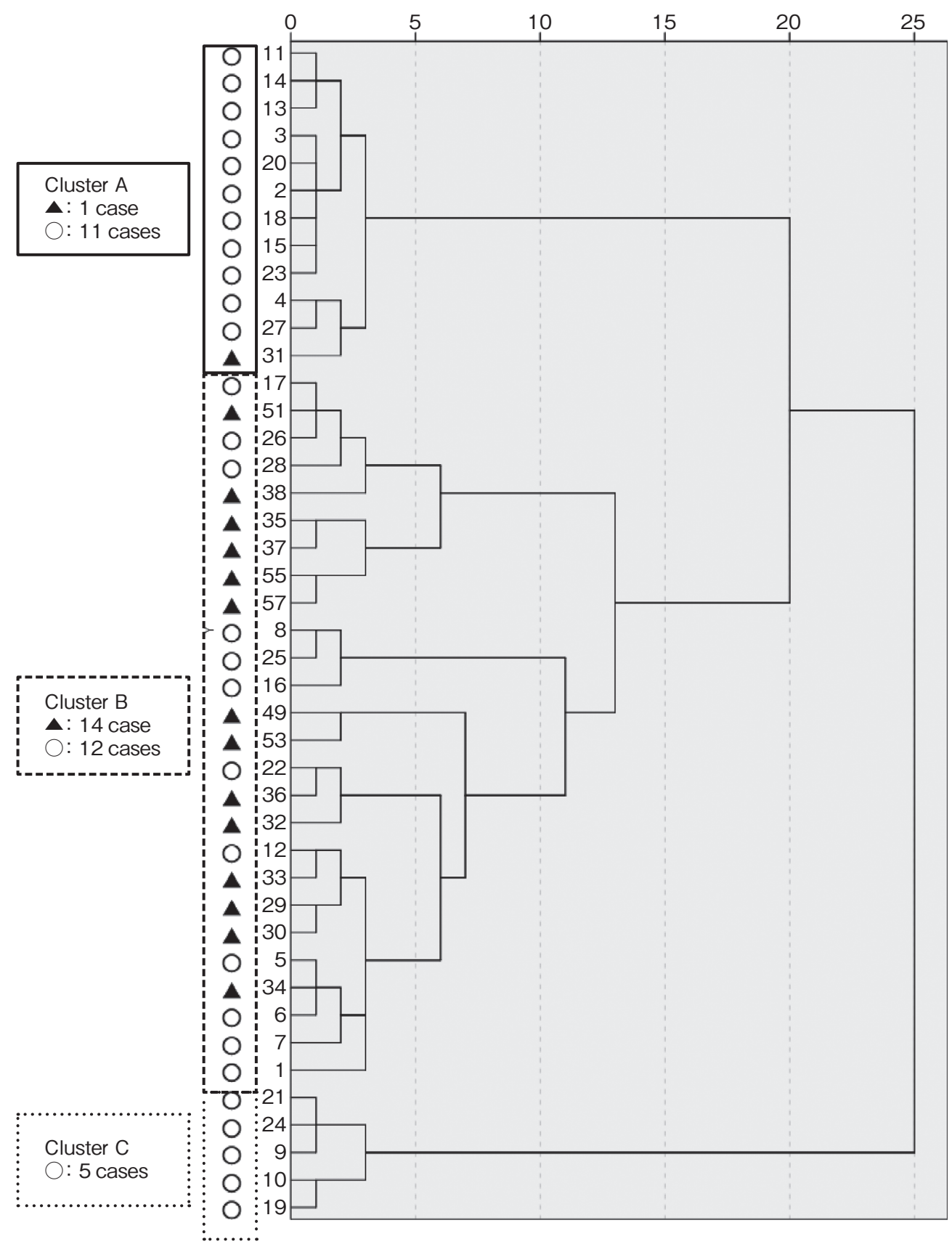

Figure-1 Analysis of fecal bacterial communities from all participants

The dendrogram shows the similarities of intestinal microbiota terminal restriction fragment length polyphism (T-RFLP) patterns from 43 fecal samples. The data from T-RFLP analysis used the restriction enzyme BslI. Solid triangles $(\boldsymbol{\Delta})$ represent colonic diverticulosis, and open circles $(\bigcirc)$ represent healthy samples. Eleven of 28 healthy individuals (39\%) were included in cluster A.

volunteers, 23 men and five women, mean age of 66.5 years [range: $54-81$ years], without constipation, colon diverticula by colonoscopy, or serious disease were collected as control samples. None of the control participants had been taking any intestinal regulators or received any oral antibiotics within the past one month. Although a few control participants had diabetes mellitus or hypertension, their conditions were being controlled with medication.

The dendrogram shows a comparison between fecal microbial communities in Group I and Group II. The data are from cluster analyses of fecal microbial communities from BslI-digested T-RF patterns (Figure-1). T-RF patterns were assigned to three major clusters (clusters A, B and C) on the 
Table-1 Terminal restriction fragment length polymorphism profiling of fecal samples from healthy individuals and those with diverticular disease

\begin{tabular}{llcc}
\hline OTU & \multicolumn{1}{c}{ Genus } & $\begin{array}{c}\text { Group I } \\
(\mathrm{n}=28)\end{array}$ & $\begin{array}{c}\text { Group II } \\
(\mathrm{n}=15)\end{array}$ \\
\hline 106 & $\begin{array}{l}\text { Clostridium subcluster } \\
\text { XIVa }\end{array}$ & $2.53 \pm 0.92$ & $0.37 \pm 0.19^{*}$ \\
\hline 317 & Prevotella & $6.83 \pm 2.49$ & $0.42 \pm 0.42^{*}$ \\
\hline 369 & Clostridium cluster IV & $0.41 \pm 0.41$ & $3.15 \pm 1.22^{*}$ \\
\hline 657 & Lactobacillales & $5.01 \pm 1.58$ & $12.56 \pm 2.65^{*}$ \\
\hline 749 & Clostridium cluster IV & $5.76 \pm 0.95$ & $1.00 \pm 0.52^{* *}$ \\
\hline 919 & $\begin{array}{l}\text { Clostridium cluster XI } \\
\text { Clostridium subcluster } \\
\text { XIVa }\end{array}$ & $1.52 \pm 0.26$ & $2.72 \pm 0.40^{*}$ \\
\hline 940 & $\begin{array}{l}\text { Clostridium subcluster } \\
\text { XIVa } \\
\text { Enterobacteriales }\end{array}$ & $5.27 \pm 0.61$ & $13.99 \pm 3.08^{*}$ \\
\hline 955 & $\begin{array}{l}\text { Clostridium } \text { subcluster } \\
\text { XIVa }\end{array}$ & $5.06 \pm 0.51$ & $2.31 \pm 0.76^{* *}$ \\
\hline
\end{tabular}

Data are mean \pm standard deviation of the percentage of the total area under the curve (\%AUC). OTU, operational taxonomic unit. ${ }^{\text {a) }}$ Group I: stool samples from healthy individuals. ${ }^{\text {b) }}$ Group II: fecal samples collected from the diverticula of patients with colonic diverticulosis.

${ }^{*} \mathrm{p}<0.05,{ }^{* *} \mathrm{p}<0.01$

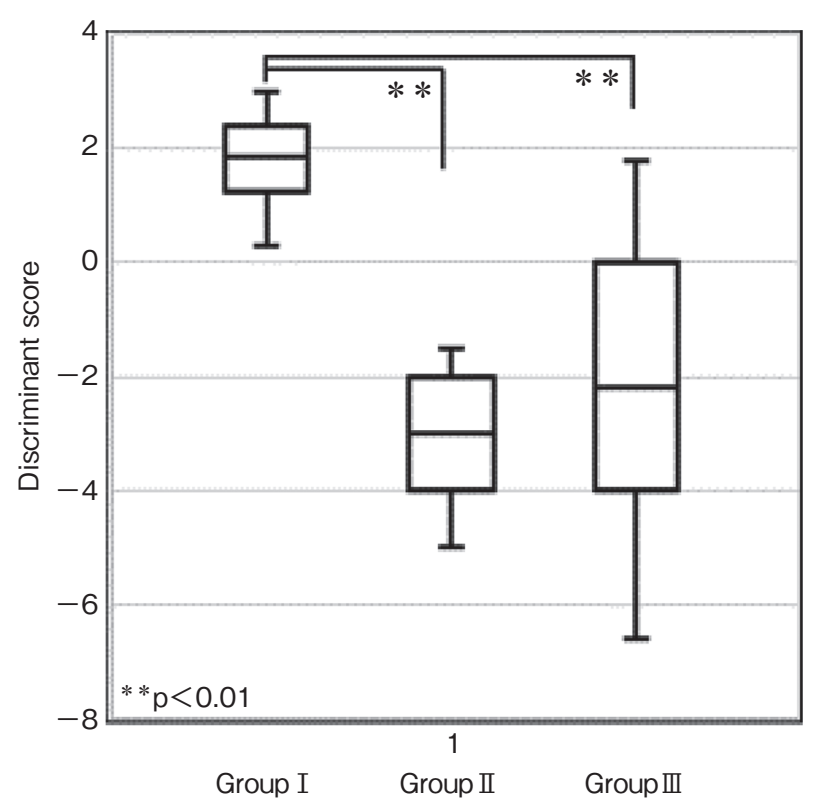

Figure-2 Box plot comparing the diversity of terminal restriction fragment length polymorphism profiles in fecal microbiota between the three groups The canonical judgment function coefficient was obtained from discriminant analysis.

basis of their similarities. Of the 28 healthy individuals, 11 were in cluster A, 12 were in cluster $\mathrm{B}$, and five were in cluster $\mathrm{C}$. For the 15 patients with diverticular disease, T-RF patterns from 14 of 15 samples from Group II were in cluster B, and the remaining one in cluster $\mathrm{A}$. The \%AUC values of representative OTUs after $B s l$ digestion are shown in Table-1. The OTUs $(369,657,919,940 \mathrm{bp})$ were increased in Group II. In particular, OTUs of $657 \mathrm{bp}$ (Lactobacillales) and $940 \mathrm{bp}$ (Clostridium subcluster XIVa, Enterobacteriales) were significantly increased in Group II. In contrast, OTUs (106, 317, 749 , and $955 \mathrm{bp)}$ predicting the Clostridium genus were decreased in Group II compared with Group I, with a significant decrease in OTUs of $749 \mathrm{bp}$ (Clostridium cluster IV) and $955 \mathrm{bp}$ (Clostridium subcluster XIVa). The canonical judgment function coefficient was obtained from discriminant analysis of Groups I, II and III. The results showed that the scores of Groups II and III were significantly different from those of Group I using one-way ANOVA (both $\mathrm{p}<0.01$ ). There were no significant differences between Groups II and III (Figure-2).

OTUs were then compared among the three groups using one-way ANOVA. An OTU of $657 \mathrm{bp}$ (Lactobacillales) was increased in Groups II and III compared with Group I $(\mathrm{p}<0.05)$. Additionally an OTU of $955 \mathrm{bp}$ (Clostridium subcluster XIVa) was decreased in Groups II and III compared with Group I $(\mathrm{p}<0.01$, Figure-3). 

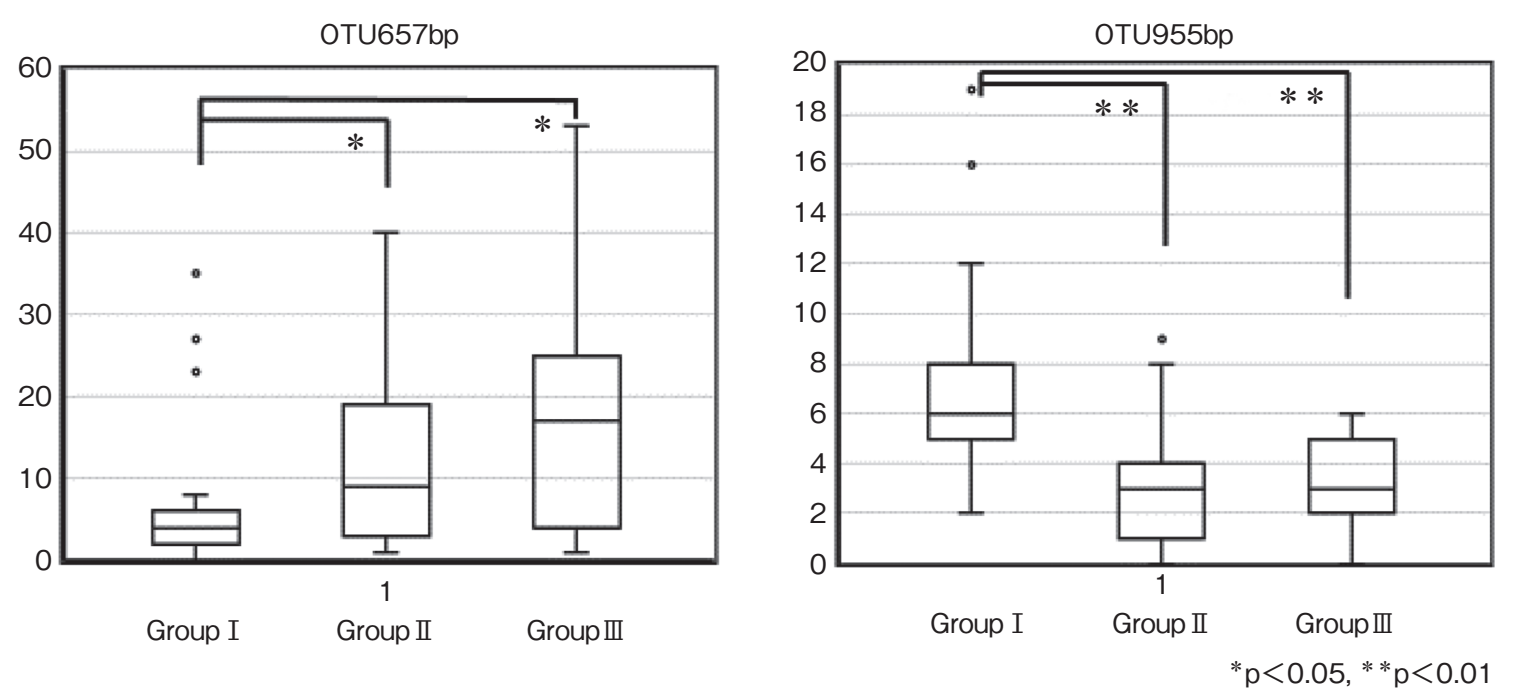

Figure-3 Analysis of operational taxonomic units (OTU) in stool samples from the three groups

\section{Discussion}

The prevalence of colonic diverticulitis has increased worldwide. In Japan, diverticula were located predominantly in the right side of the colon in $50.0 \%$ of cases, bilaterally in $29.3 \%$ of cases, and in the left side in $20.7 \%$ of cases $^{10}$.

The prevalence of colonic diverticulosis increases with age. In this pilot study, we focused on the right diverticulum, which is the most common location of diverticulosis in the Japanese population. In our follow-up studies, we will examine the left diverticulum.

The pathophysiology of colon diverticulitis is complex and may be related to genetic factors, abnormal colonic motility, abnormality in the autonomic nervous system, macroscopic morphological changes in the large intestine, visceral hypersensitivity, and an imbalance in colonic microflora $^{2)}$. To our knowledge, this study is the first to show an altered profile of intestinal microbiota in feces accumulated in the diverticula. Information on microbial diversity in patients with diverticular disease is relatively scarce. Here, we utilized a T-RFLP approach to compare microbial diversity in gastrointestinal populations from patients with diverticular disease and healthy individuals.

T-RFLP analysis facilitates the comparison of complex microbiota diversity, because the fluorescently labeled T-RFs are able to be visualized ${ }^{11)}{ }^{12}$. Notably, no studies have shown whether the fecal microbiota communities accumulated in the diverticula differ from those of stool specimens in the same patients. If the fecal matter accumulated in the diverticula of a patient with diverticulitis and the microbiota of the patient's natural defecation were the same, it could be assumed that microbiota analysis would be easier. Our findings suggest that there were no differences in these communities. Moreover, our cluster analysis dendrogram indicates that the fecal microbial communities of patients with diverticular disease differed from those of healthy volunteers. These differences involved a significant decrease in the Clostridium genus, including Clostridium cluster IV and subcluster XIVa, and a significant increase in Lactobacillales and Enterobacteriales in patients with diverticular disease. Decreased Clostridium spp. is a common feature in many studies of inflammatory bowel disease (IBD) ${ }^{13)}{ }^{14}$. Linninge et al. demonstrated that patients with diverticular disease had higher amounts of Enterobacteriaceae in the colon mucosa than patients without diverticula ${ }^{15)}$. However, it is still unclear whether diverticulitis occurs due to changes in the gut microbiota. Spiller and Sloan hypothesized that the presence of diverticula generates a unique environment that contributes to important changes in the diversity of fecal microorganisms with potential effects on the intestinal immune system ${ }^{16)}$. The differences between the feces of patients with diverticular disease and those of healthy individuals suggest that dysbiosis contributes to diverticular disease. 
In this study, we focused on two OTUs (657 and $955 \mathrm{bp})$. In patients with diverticular disease, we found that an OTU of $657 \mathrm{bp}$ (Lactobacillales) was increased, whereas that of $955 \mathrm{bp}$ (Clostridium subcluster XIVa) was decreased. The OTU of 657 bp included Streptococcus spp. (S. parasanguinis, S. salivarius, S. thermophilus, S. vestibularis, S. pasteuri), and Lactobacillus spp. (L. salivarius, L. cellobiosus). Lactobacillus is a beneficial microbial genus, acting to prevent intestinal infection and activate the immune system. Thus, it seems counter-intuitive that Lactobacillus was increased in patients with diverticular disease. On the other hand, it has been reported that Lactobacillus is increased in patients with IBS characterized by abnormal bowel movements ${ }^{9}$. Streptococcus, however, may increase inflammation in the human gut. Little is known about Clostridium subcluster XIVa, with the exception that it contains many species that produce butyric acid. Butyrate has many beneficial effects in the host such as trophic and anti-inflammatory effects on epithelial cells ${ }^{17)}$. Thibault et al. showed that the butyrate transporter is down-regulated in the colonic mucosa of patients with $\mathrm{IBD}^{18)}$. In addition, butyrate-producing bacteria are decreased in the gut microorganisms of the intestinal mucosa and feces of patients with $\mathrm{IBD}^{19)}$. Furusawa et al. demonstrated that butyrate induces the differentiation of colonic regulatory $\mathrm{T}$ cells in mice ${ }^{20)}$. Clostridia can induce colonic regulatory $\mathrm{T}$ cells, which are involved in the suppression of inflammatory and allergic responses ${ }^{21)}$. These results suggest that an insufficiency of butyrate may be associated with the pathogenesis of IBD. Similarly, decreases in Clostridium subcluster XIVa found in patients with diverticular disease may result in a reduction in butyric acid in the bowel, which may contribute to diverticular disease development.

This study had some limitations. Firstly, we did not control for sex, medication, or diet; instead, patients with stomachache were subjected to colonoscopy, which may have led to selection bias. In our follow-up studies, we will control for subject characteristics such as sex, medication, and localization of diverticulitis in the colon. Secondly, the number of patients was small as this was only a pilot study. Additional studies are needed to confirm our findings. Thirdly, although T-RFLP analysis pro- vides reproducible comparisons of microbial communities $^{6)}$, it does not provide high-resolution data, as does NGS. Finally, this study was performed at a single institution. Recent studies have demonstrated that regional characteristics affect the fecal microbiota; thus, our observations may be limited to the region in which our hospital is located ${ }^{22)}$ 23).

In conclusion, T-RFLP analyses revealed that the fecal microbial communities in patients with diverticular disease differed from those of healthy individuals, particularly for the operational taxonomic units of 657 and $955 \mathrm{bp}$. Changes in the fecal microbiota, including Lactobacillales and Clostridium subcluster XIVa, may play a role in diverticular disease.

\section{Conflict of interest}

The authors declare no conflicts of interest in association with the present study.

\section{References}

1) Sheth AA, Longo W, Floch MH: Diverticular disease and diverticulitis. Am J Gastroenterol, 2008; 103: 15501556.

2) Tursi A: Diverticular disease: A therapeutic overview. World J Gastrointest Pharmacol Ther, 2010; 1: 27-35.

3) Stollman NH, Raskin JB: Diagnosis and management of diverticular disease of the colon in adults. Am J Gastroenterol, 1999; 94: 3110-3121.

4) Papi C, Ciaco A, Koch M, Capurso L: Efficacy of rifaximin in the treatment of symptomatic diverticular disease of the colon. A multicentre double-blind placebo-controlled trial. Aliment Pharmacol Ther, 1995; 9: 33-39.

5) Latella G, Pimpo MT, Sottili S, et al: Rifaximin improves symptoms of acquired uncomplicated diverticular disease of the colon. Int J Colorectal Dis, 2003; 18: 55-62.

6) Andoh A, Tsujikawa T, Sasaki M, et al: Faecal microbiota profile of Crohn's disease determined by terminal restriction fragment length polymorphism analysis. Aliment Pharmacol Ther, 2009; 29: 75-82.

7) Nagashima K, Mochizuki J, Hisada T, Suzuki S, Shimomuraet K: Phylogenetic analysis of $16 \mathrm{~S}$ ribosomal RNA gene sequences from human fecal microbiota and improved utility of terminal restriction fragment length polymorphism profiling. Biosci Microflora, 2006; 25: 99107.

8) Nagashima K, Hisada T, Sato M, Mochizuki J: Application of new primer-enzyme combinations to terminal restriction fragment length polymorphism profiling of bacterial populations in human feces. Appl Environ Microbiol, 2003; 69: 1251-1262.

9) Tana C, Umesaki Y, Imaoka A, Handa T, Kanazawa M, Fukudo S: Altered profiles of intestinal microbiota and organic acids may be the origin of symptoms in irritable bowel syndrome. Neurogastroenterol Motil, 2010; 22: 
512-519.

10) Nagata N, Niikura R, Shinmbo T, et al: Alcohol and smoking affect risk of uncomplicated colonic diverticulosis in Japan. PLoS One, 2013; 8: e81137.

11) Liu WT, Marsh TL, Cheng H, Forney LJ: Characterization of microbial diversity by determining terminal restriction fragment length polymorphisms of genes encoding 16S rRNA. Appl Environ Microbiol, 1997; 63: 4516-4522.

12) Sakamoto M, Hayashi H, Benno Y: Terminal restriction fragment length polymorphism analysis for human fecal microbiota and its application for analysis of complex bifidobacterial communities. Microbiol Immunol, 2003; 47: 133-142.

13) Swidsinski A, Ladhoff A, Pernthaler A, et al: Mucosal flora in inflammatory bowel disease. Gastroenterology, 2002; 122: 44-54.

14) Martinez-Medina M, Aldeguer $X$, Gonzalez-Huix F, Acero D, Garcia-Gil LJ: Abnormal microbiota composition in the ileocolonic mucosa of Crohn's disease patients as revealed by polymerase chain reaction-denaturing gradient gel electrophoresis. Inflamm Bowel Dis, 2006; 12: $1136-1145$

15) Linninge C, Roth B, Erlanson-Albertsson C, Molin G, Toth E, Ohlsson B: Abundance of Enterobacteriaceae in the colon mucosa in diverticular disease. World $\mathrm{J}$ Gastrointest Pathophysiol, 2018; 9: 18-27.

16) Spiller RC, Sloan TJ: Do diverticula provide a unique niche for microbiota which can lead to activation of the innate immune system? Gut, 2017; 66: 1175-1176.

17) Inan MS, Rasoulpour RJ, Yin L, Hubbard AK, Rosenberg DW, Giardina C: The luminal short-chain fatty acid butyrate modulates $\mathrm{NF}-\kappa \mathrm{B}$ activity in a human colonic epithelial cell line. Gastroenterology, 2000; 118: 724-734.

18) Thibault R, De Coppet $P$, Daly K, et al: Down-regulation of the monocarboxylate transporter 1 is involved in butyrate deficiency during intestinal inflammation. Gastroenterology, 2007; 133: 1916-1927.

19) Frank DN, St Amand AL, Feldman RA, et al: Molecular-phylogenetic characterization of microbial community imbalances in human inflammatory bowel diseases. Proc Natl Acad Sci U S A, 2007; 104: 13780-13785.

20) Furusawa $Y$, Obata $Y$, Fukuda S, et al: Commensal microbe-derived butyrate induces the differentiation of colonic regulatory T cells. Nature, 2013; 504: 446-450.

21) Atarashi $\mathrm{K}$, Tanoue $\mathrm{T}$, Shima $\mathrm{T}$, et al: Induction of colonic regulatory $\mathrm{T}$ cells by indigenous clostridium species. Science, 2011; 331: 337-341.

22) De Filippo C, Cavalieri D, Di Paola M, et al: Impact of diet in shaping gut microbiota revealed by a comparative study in children from Europe and rural Africa. Proc Natl Acad Sci U S A, 2010; 107: 14691-14696.

23) Andoh A, Kuzuoka H, Tsujikawa T, et al: Multicenter analysis of microbiota profiles in Japanese patients with Crohn's disease. J Gastroenterol, 2012; 47: 1298-1307. 